\title{
Frauen- und Ausländeranteil nehmen kontinuierlich zu
}

\author{
Stefanie Hostettlera, Esther Kraft ${ }^{\mathrm{b}}$ \\ ${ }^{a}$ Dr. Sc. ETH Zürich, Abteilung Daten, Demographie und Qualität DDQ FMH; ${ }^{b}$ lic. rer. oec., Leiterin Abteilung Daten, Demographie und Qualität DDQ FMH
}

Die FMH setzt sich seit Jahren für qualitativ hochstehende Daten ein. Die FMHÄrztestatistik fördert den Wissenstransfer und leistet einen wichtigen Beitrag zur Abschätzung der aktuellen Versorgungssituation in der Schweiz. Die Abteilung Daten, Demographie und Qualität (DDQ) publiziert jährlich die neusten Zahlen und Entwicklungen rund um das Thema Ärztestatistik.

\section{FMH-Ärztestatistik - systematische Erhebung seit 1934}

Eine bedarfsgerechte und qualitativ hochstehende Versorgung der Bevölkerung: Dieses Ziel ist handlungsleitend für Fachleute und Institutionen im Gesundheitswesen. Die hohe Behandlungsintensität in einer alternden Gesellschaft beeinflusst nebst weiteren demographischen, wirtschaftlichen und gesellschaftlichen Faktoren die Entwicklungen des Gesundheitssystems. Die zunehmende Nachfrage nach Teilzeitarbeit, die Einführung von SwissDRG, die Beschränkung von Aus- und Weiterbildungsplätzen sowie der Zulassungsstopp stellen das Schweizer Gesundheitssystem vor aktuelle und zukünftige Herausforderungen wie beispielsweise ein verschärfter Ärztemangel in manchen Fachgebieten [1]. Zwar zeigt der Ärztebestand einen jährlichen Anstieg der Anzahl berufstätiger Ärztinnen und Ärzte in der Schweiz. Ein differenzierter Blickwinkel lässt jedoch vermuten: Wenn Wohnbevölkerung, Durchschnittsalter und Teilzeitarbeit zunehmen und gleichzeitig der Anteil an Generalisten abnimmt, dann stösst die Kapazität zur Erbringung von Gesundheitsdienstleistungen an Grenzen. Vor diesem Hintergrund ist es zentral, aktuelle Entwicklungen im Gesundheitswesen $\mathrm{zu}$ verfolgen, die bevorstehenden Probleme zu erkennen und diesen gezielt entgegenzuwirken.

Die FMH-Ärztestatistik stellt seit vielen Jahren ein wichtiger Bestandteil zur Bedarfsabklärung dar. Jährlich publiziert die FMH in der Schweizerischen Ärztezeitung und auf ihrer Website die Auswertungen demographischer Merkmale, die Angaben zur Berufstätigkeit sowie weitere Angaben der Ärzteschaft.
Die Daten sind unter anderem für die Versorgungsforschung, als evidenzbasierte Entscheidungsgrundlage in der Gesundheitspolitik sowie zum Zweck der Wissensvermittlung unabdingbar. Ebenso nutzen Ärzteorganisationen und weitere Interessierte diese Daten als Informationsquelle für fachspezifische Fragestellungen.

\section{Datengrundlagen}

Die FMH-Ärztestatistik wird zum Jahresende (Stichtag 31. Dezember des jeweiligen Jahres) neu erstellt. Der Ergebnisteil der Ärztestatistik gliedert sich in zwei Teile. Der erste Teil beruht auf Angaben der FMHDatenbank ( $n=34348)$ und enthält die wichtigsten ärztedemographischen Merkmale wie das Alter, das Geschlecht, die Nationalität und den Ort der Berufsausübung. Im zweiten Teil befinden sich Ergebnisse der jährlichen Umfrage zur Berufstätigkeit (Arbeitspensum, Praxisstruktur usw.). Diese Angaben werden mittels Fragebogen auf dem Mitgliederportal myFMH erhoben und beruhen auf Selbstdeklaration (myFMHStichprobe). Die Datengrundlage beinhaltet Angaben von über 12000 Ärzten* (Rücklauf 42,3 Prozent).

\section{Repräsentativität der myFMH-Stichprobe}

Die Stichprobe und die Grundgesamtheit wurden hinsichtlich verschiedener Merkmale (Geschlecht, Sektor, Fachrichtung) miteinander verglichen. Dies diente

Weiterführende Informationen zur FMH-Ärztestatistik und zur Mitgliederstatistik sowie zu den wichtigsten Indikatoren finden Sie auf der Website der FMH (www.fmh.ch $\rightarrow$ Services $\rightarrow$ Statistik). Gerne steht Ihnen die Abteilung DDQ für weiterführende Auswertungen und Fragen zur Verfügung. Kontakt: ddq[at]fmh.ch / 0313591111. 
der Überprüfung, ob es zulässig ist, auf der Grundlage der vorhandenen Daten aus dem myFMH-Fragebogen ( $n=12364)$ Rückschlüsse von der Stichprobe auf die Grundgesamtheit der berufstätigen Ärzte in der Schweiz zu ziehen. Die Häufigkeit der oben aufgeführten Merkmale in der Stichprobe sind weitgehend mit derjenigen aus der Grundgesamtheit vergleichbar (Differenz <10 Prozent). Einzig der stationäre Sektor und Frauen im stationären Sektor sind in der Stichprobe untervertreten. Um diese Verzerrung aufzuheben, sind die Auswertungen nach Sektor und Geschlecht getrennt erfolgt oder entsprechend gewichtet. In vorangehenden Studien bestätigt der Vergleich der selbstdeklarierten Angaben zum Arbeitspensum mit den individuell abgerechneten TARMED-Leistungen bzw. den hinterlegten Minutagen eine gute Übereinstimmung [2-4].

\section{Ärzteschaft - Männer (noch) in der Überzahl}

Die Zahl der berufstätigen Ärzte ist weiter angestiegen (Abb. 1). Im Jahr 2014 waren 34348 Ärzte (13589 Frauen, 20759 Männer) in der Schweiz berufstätig, 1106 mehr als im Vorjahr. Nach wie vor sind die Männer $(60,4$ Prozent) gegenüber ihren Kolleginnen (39,6 Prozent) in der Überzahl. Der Frauenanteil ist hingegen in den letzten Jahren im Vergleich zu den Männern immer mehr gestiegen. Während seit 1990 die jährliche Zunahme des Frauenanteils im Durchschnitt 4,8 Prozent betrug, ist sie bei den Männern lediglich 1,2 Prozent. Auch die seit mehreren Jahren beobachtete Entwicklung des Geschlechterverhältnisses bei der Aus- und Weiterbildung von Ärzten zeigt, dass der Frauenanteil der berufstätigen Ärzteschaft in den kommenden Jahren weiter zunehmen wird. Bei den Bildungsabschlüssen in der Humanmedizin überwiegt der Frauenanteil bereits seit zehn Jahren - aktuell liegt er bei 61,9 Prozent (Frauen 518, Männer 318) [5]. Bei den Erteilungen der eidgenössisch anerkannten Facharzttitel (1501 total) beträgt dieser 54,2 Prozent. Durchschnittlich arbeiten 4,1 Ärzte pro 1000** Einwohner in der Schweiz, was im internationalen Vergleich über dem Durchschnitt liegt (2013: 3,7 Ärzte

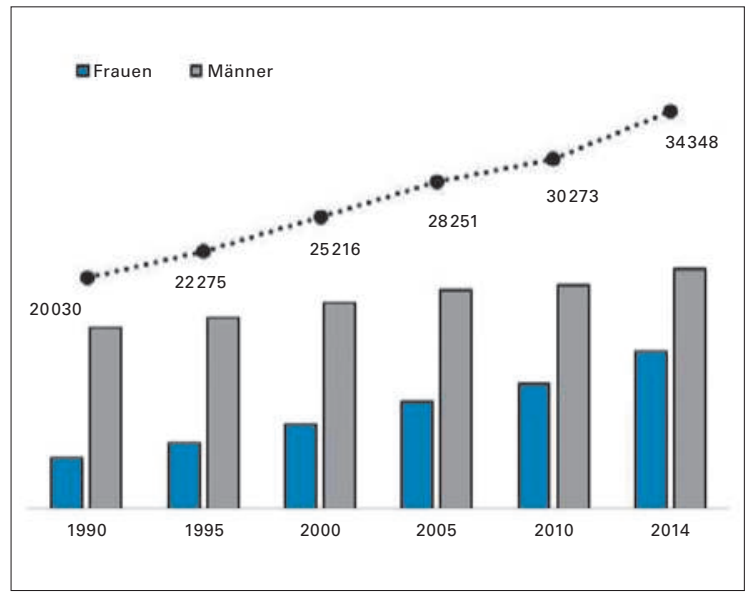

Abbildung 1: Anzahl Ärzte nach Jahr und Geschlecht 1990-2014.

pro 1000 Einwohner) [6]. Die Schweizer Kantone mit den höchsten Dichten an Ärzten sind Basel-Stadt (9,7 Ärzte pro 1000 Einwohner), Genf $(6,2)$ und Zürich $(4,9)$. Die tiefsten Ärztedichten weisen dagegen Uri (1,6 Ärzte pro 1000 Einwohner), Appenzell IR $(1,6)$ und Nidwalden $(2,2)$ auf.

\section{Arbeitsort - ambulanter, stationärer und anderer Sektor}

Die Mehrheit der Ärzte (51,8 Prozent) ist hauptberuflich im ambulanten Sektor tätig (Tab. 1). 46,5 Prozent arbeiten im stationären und 1,7 Prozent in einem anderen Sektor (Versicherungen, Verbände usw.). Im ambulanten Bereich stieg die Anzahl Ärzte im Vergleich zum Vorjahr um 1,4 Prozent, im stationären Bereich um 5,6 Prozent. Ebenfalls erhöht hat sich die Anzahl der Ärzte, welche in einem anderen Bereich arbeiten (z.B. Verwaltung, Versicherungen) (o,4 Prozent). Abbildung 2 verdeutlicht, dass Ärzte mit zunehmendem Alter vermehrt im ambulanten Bereich tätig sind.

Der Frauenanteil ist im stationären Bereich (44,5 Prozent) höher als im ambulanten Sektor (35,5 Prozent). Ärzte in der Schweiz arbeiten hauptsächlich in einem Sektor (89,5 Prozent), ein kleiner Teil der Ärzteschaft ist in mehreren Sektoren tätig (10,4 Prozent). Davon

\footnotetext{
** Die Angaben zur Ärztedichte gelten für das Jahr 2013, da die Bevölkerungszahlen des Bundesamts für Statistik (BFS) zum Zeitpunkt der Publikation der Ärztestatistik noch nicht verfügbar sind.
}

Tabelle 1: Übersicht der berufstätigen Ärzte nach Sektoren (Hauptberufstätigkeit) 2014.

\begin{tabular}{|c|c|c|c|c|c|c|}
\hline \multicolumn{3}{|c|}{ Frauen } & \multicolumn{2}{|c|}{ Männer } & \multicolumn{2}{|l|}{ Total } \\
\hline Ambulanter Sektor & 6313 & $35,5 \%$ & 11491 & $64,5 \%$ & 17804 & $51,8 \%$ \\
\hline Stationärer Sektor & 7105 & $44,5 \%$ & 8876 & $55,5 \%$ & 15981 & $46,5 \%$ \\
\hline Total & 13589 & $39,6 \%$ & 20759 & $60,4 \%$ & 34348 & $100,0 \%$ \\
\hline
\end{tabular}




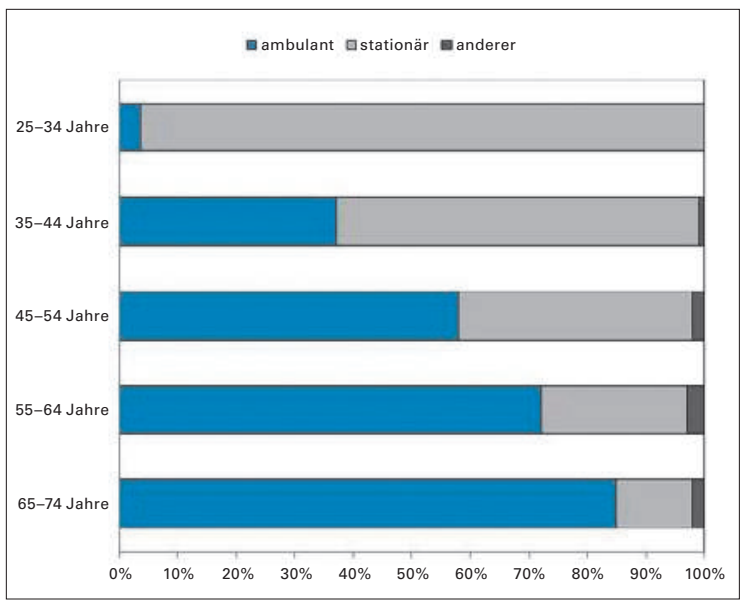

Abbildung 2: Arbeitsort nach Altersklasse 2014.

arbeitet der grösste Teil (82,5 Prozent) hauptberuflich im ambulanten Sektor und führt zusätzlich eine Nebentätigkeit im stationären Bereich aus, zum Beispiel als Belegärzte.

\section{Altersstruktur - Frauen unter 35 Jahre in der Überzahl}

Das Durchschnittsalter der Ärzte in der Schweiz im Jahr 2014 beträgt 48,9 Jahre (Tab. 2). Dieses nimmt seit Jahren zu und lag vor einem Jahrzehnt noch bei 45,8 Jahren. Ärzte im ambulanten Sektor (54,o Jahre) sind durchschnittlich zehn Jahre älter als ihre Kollegen im stationären Sektor (42,9 Jahre). Dieser Altersunterschied zwischen den Sektoren ist hauptsächlich durch die Weiterbildung der Assistenzärzte bedingt, welche vorwiegend in Spitälern absolviert wird. Im Jahr 2014 waren die Ärzte zum Abschluss der Weiter- bildung bzw. Erwerb des ersten Facharzttitels durchschnittlich 37,0 Jahre alt (Frauen 36,5 Jahre; Männer 37,7 Jahre).

Aus Abbildung 3 wird ersichtlich, dass Frauen in der Altersklasse unter 35 Jahren deutlich in der Überzahl sind. Ab dem 40. Lebensjahr sind zurzeit mehr Männer als Frauen berufstätig. Aufgrund der Frauenmehrheit bei den Studierenden und bei der jungen Ärzteschaft ist davon auszugehen, dass sich der wachsende Frauenanteil in den kommenden Jahren entsprechend in der geschlechtsspezifischen Altersstruktur widerspiegeln wird.

\section{Fachrichtung - Allgemeine Innere Medizin an der Spitze}

Das Verhältnis der Generalisten (Allgemeine Innere Medizin, Kinder- und Jugendmedizin sowie Praktischer Arzt) zu den Spezialisten beträgt 37,4 zu 62,6 Prozent. Das am häufigsten vertretene medizinische Fachgebiet ist die Allgemeine Innere Medizin (23,5 Prozent). An zweiter Stelle liegt die Psychiatrie und Psychotherapie (10,3 Prozent), gefolgt von der Gynäkologie und Geburtshilfe (4,8 Prozent), Kinderund Jugendmedizin (4,8 Prozent) und die Anästhesiologie (4,2 Prozent).

Tabelle 2: Durchschnittsalter (in Jahren) der Ärzteschaft 2014

\begin{tabular}{llll} 
& Frauen & Männer & Alle \\
\hline Ambulanter Sektor & 50,9 & 55,7 & 54,0 \\
\hline Stationärer Sektor & 39,6 & 45,5 & 42,9 \\
\hline Alle & 45,0 & 51,4 & 48,9 \\
\hline
\end{tabular}

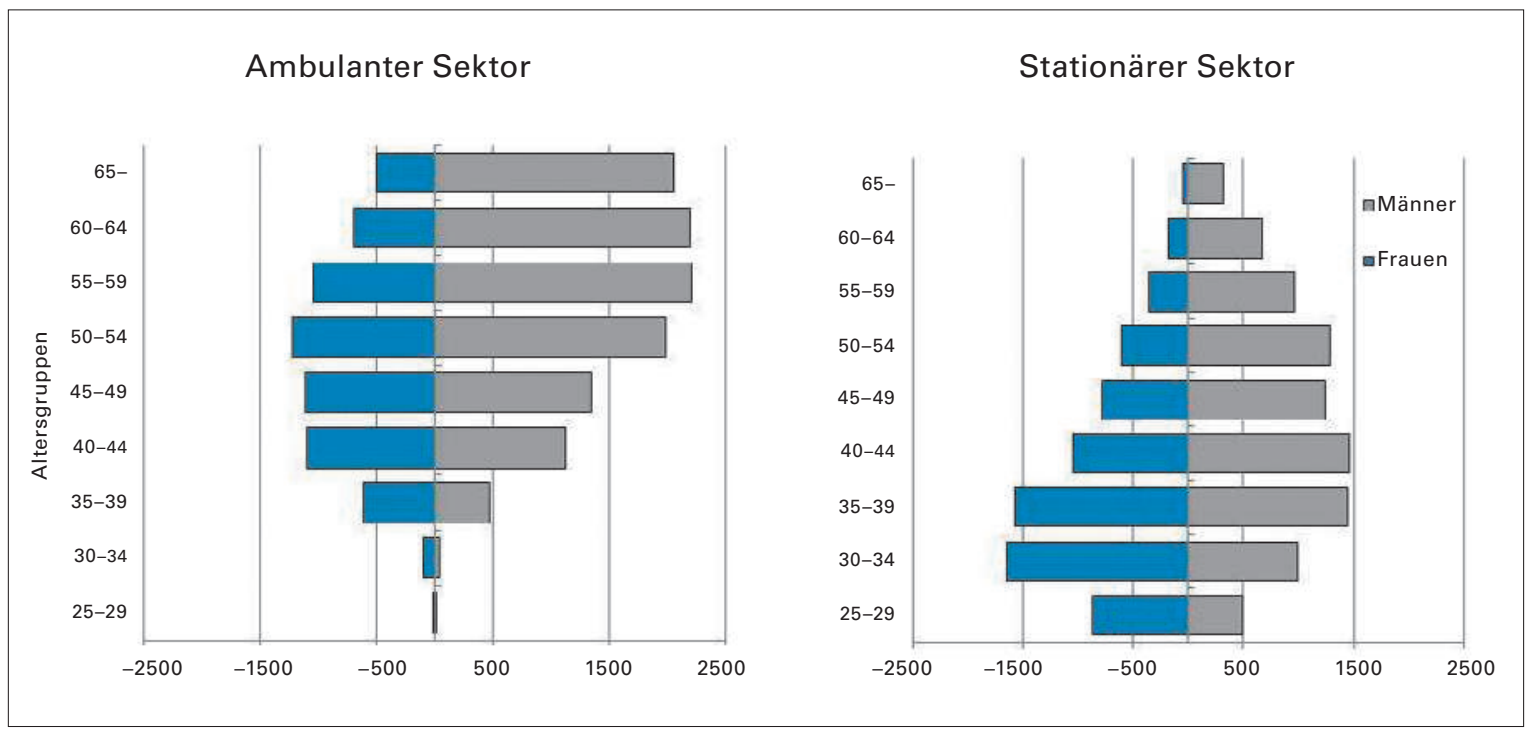

Abbildung 3: Geschlechtsspezifische Altersstruktur der Ärzte nach Sektor 2014. 
Der Frauenanteil ist in den Fachrichtungen Kinderund Jugendpsychiatrie (62,9 Prozent), Kinder- und Jugendmedizin (57,4 Prozent) und Gynäkologie und Geburtshilfe (56,6 Prozent) am höchsten. Die Männer sind im Vergleich zu den Frauen in den chirurgischen Fachgebieten in der Überzahl (Herz- und thorakale
Gefässchirurgie 92,o Prozent, Orthopädische Chirurgie 91,5 Prozent, Neurochirurgie 86,1 Prozent, Chirurgie 82,3 Prozent).

4571 Ärzte - davon 1168 Frauen - sind Mehrfachtitelträger. Der am häufigsten vertretene Facharzttitel unter den Mehrfachtitelträgern ist der Titel Allge-

Tabelle 3. Übersicht der Ärzte nach Fachrichtung (Hauptberufstätigkeit) 2014.

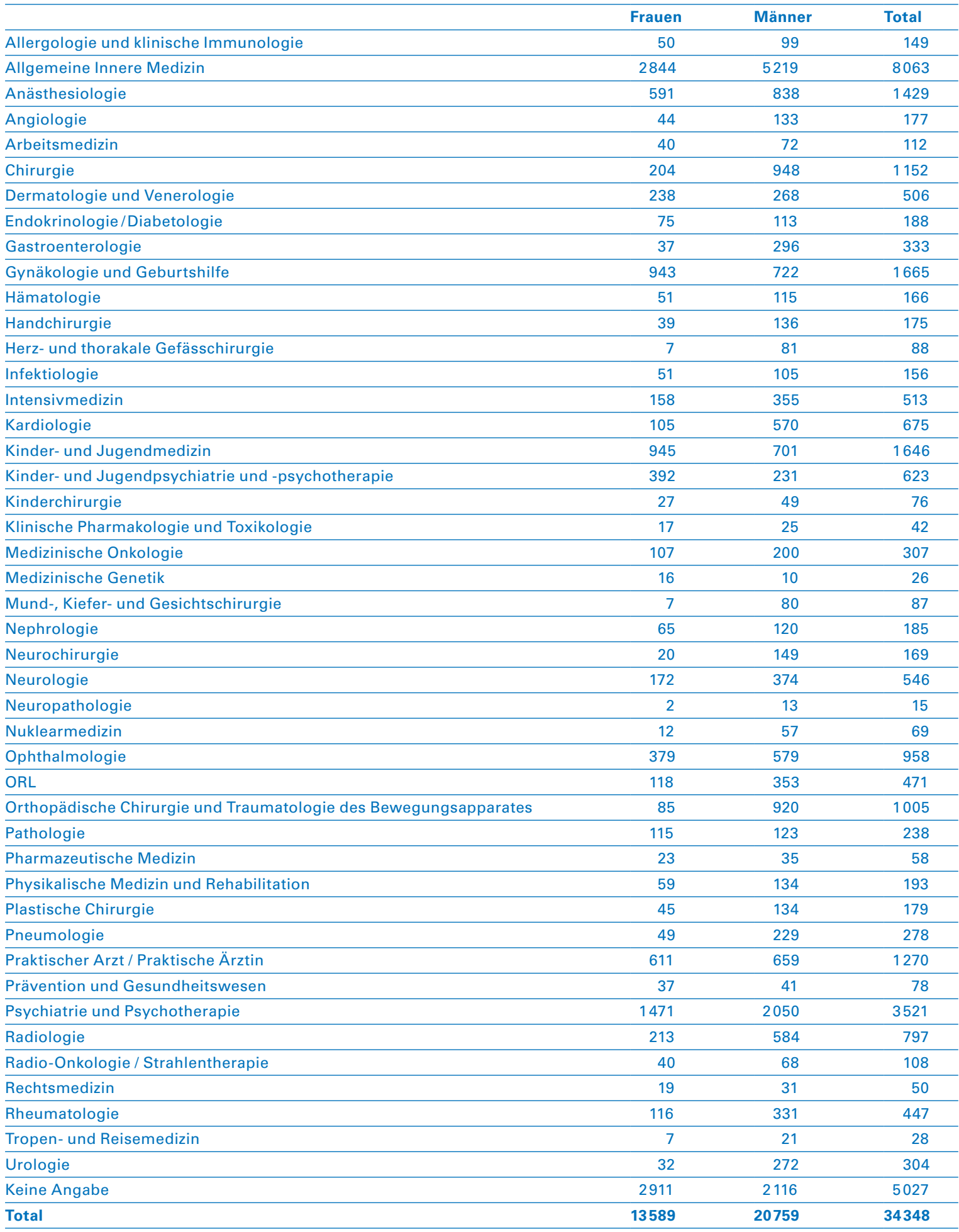


meine Innere Medizin (3277). Mehrfachtitelträger sind vorwiegend in der Kardiologie (545), Intensivmedizin (493) und Rheumatologie (383) tätig.

\section{Funktion - abnehmender Frauenanteil nach Hierarchiestufe}

Von den 17804 Ärzten im ambulanten Sektor sind 83,6 Prozent Praxisinhaber oder -teilhaber. 4,0 Prozent sind als Praxisassistent oder -facharzt angestellt. Den restlichen Ärzten kommt eine andere Funktion zu. Im stationären Sektor bilden die Assistenten in Weiterbildung mit rund 50 Prozent die grösste Gruppe. Erwartungsgemäss reduziert sich die Anzahl Ärzte mit steigender Hierarchiestufe. So sind im stationären Sektor 19,5 Prozent als Oberarzt, 12,3 Prozent als Leitender Arzt und 9,7 Prozent als Chefarzt tätig. Einzig bei den Assistenzärzten überwiegt der Frauenanteil (57,4 Prozent), danach nimmt er laufend ab: bei den Oberärzten 43,7 Prozent, bei den Leitenden Ärzten 22,4 Prozent und bloss 10,9 Prozent bei den Chefärzten.

\section{Ausländische Arztdiplome - vorwiegend aus Deutschland}

Aus Abbildung 4 wird ersichtlich, dass mit 30,5 Prozent fast ein Drittel (10 478) der berufstätigen Ärzte in
Tabelle 4: Durchschnittliches Arbeitspensum in Halbtagen pro Woche nach Sektor und Geschlecht 2014 ( $n=7295)$.

\begin{tabular}{llcl} 
& Frauen & Männer & Total \\
\hline Ambulanter Sektor & 6,8 & 8,9 & 8,2 \\
\hline Stationärer Sektor & 8,8 & 10,1 & 9,4 \\
\hline Anderer Sektor & 7,2 & 8,9 & 8,3 \\
\hline Total & 7,4 & 9,3 & 8,9 \\
\hline
\end{tabular}

der Schweiz aus dem Ausland stammen (bzw. sind Inhaber eines ausländischen Studienabschlusses der Humanmedizin). Der Ausländeranteil in der Ärzteschaft fällt je nach Grossregion sehr unterschiedlich aus (Abb. 4) und reicht von 24,1 Prozent in der Genferseeregion bis zu 46,3 Prozent im Tessin. Im Vergleich zum Vorjahreswert ist der Anteil um 1,4 auf 30,5 Prozent gestiegen. Davon sind 56,2 Prozent im stationären, 41,7 Prozent im ambulanten und 2,1 Prozent in einem anderen Bereich tätig. Die Mehrheit der Fachkräfte aus dem Ausland stammt aus Deutschland (17,4 Prozent), Italien (2,4 Prozent), Frankreich (1,7 Prozent) oder Österreich (1,7 Prozent). Mit 44 Prozent ist der Anteil an ausländischen Ärztinnen etwas tiefer als der Männeranteil (56 Prozent).

Von den insgesamt 1501 Verleihungen für Facharzttitel im Jahr 2014 gingen 44 Prozent an Ärzte mit einem ausländischen Arztdiplom.

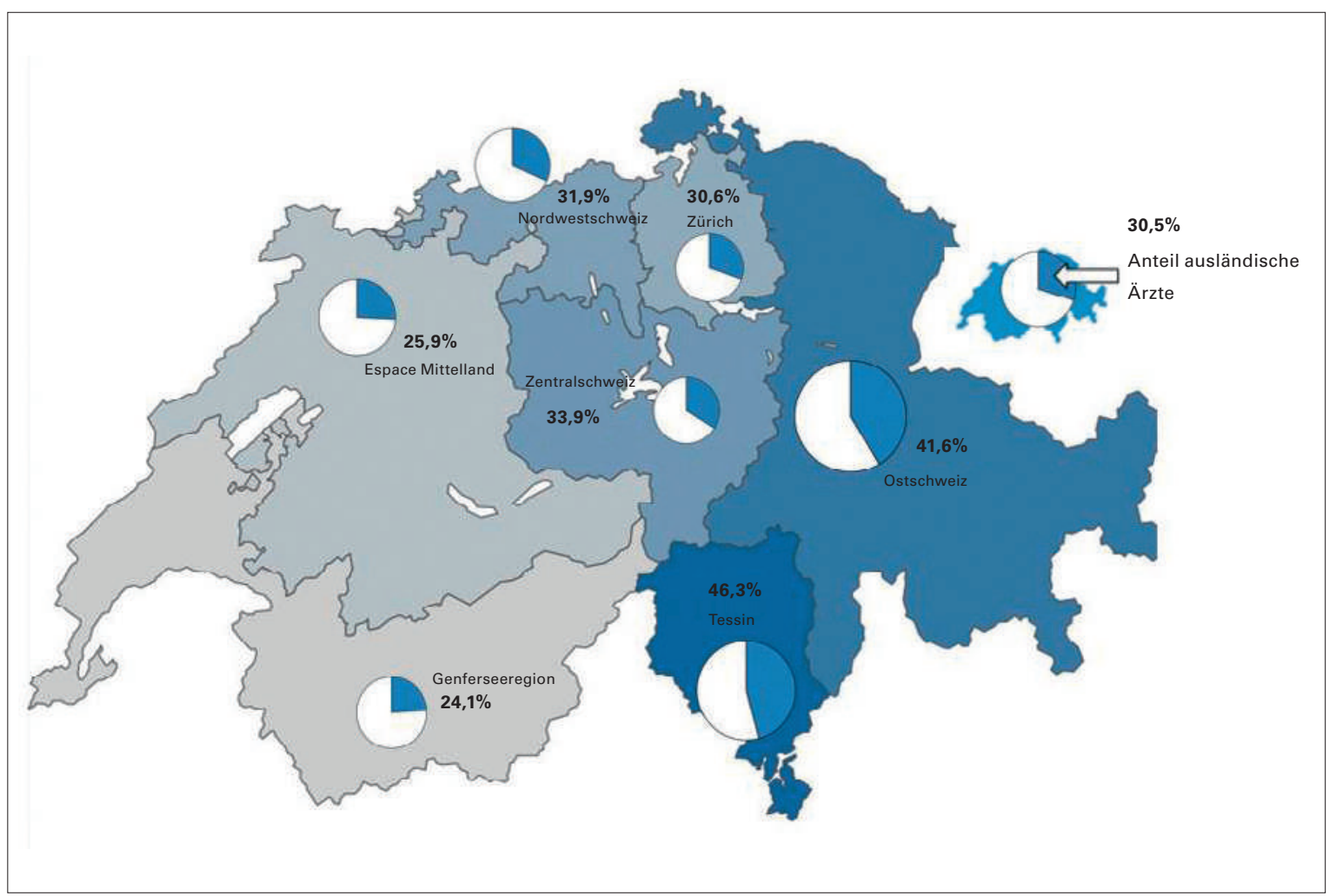

Abbildung 4: Anteil Ausländer nach Grossregion 2014. 


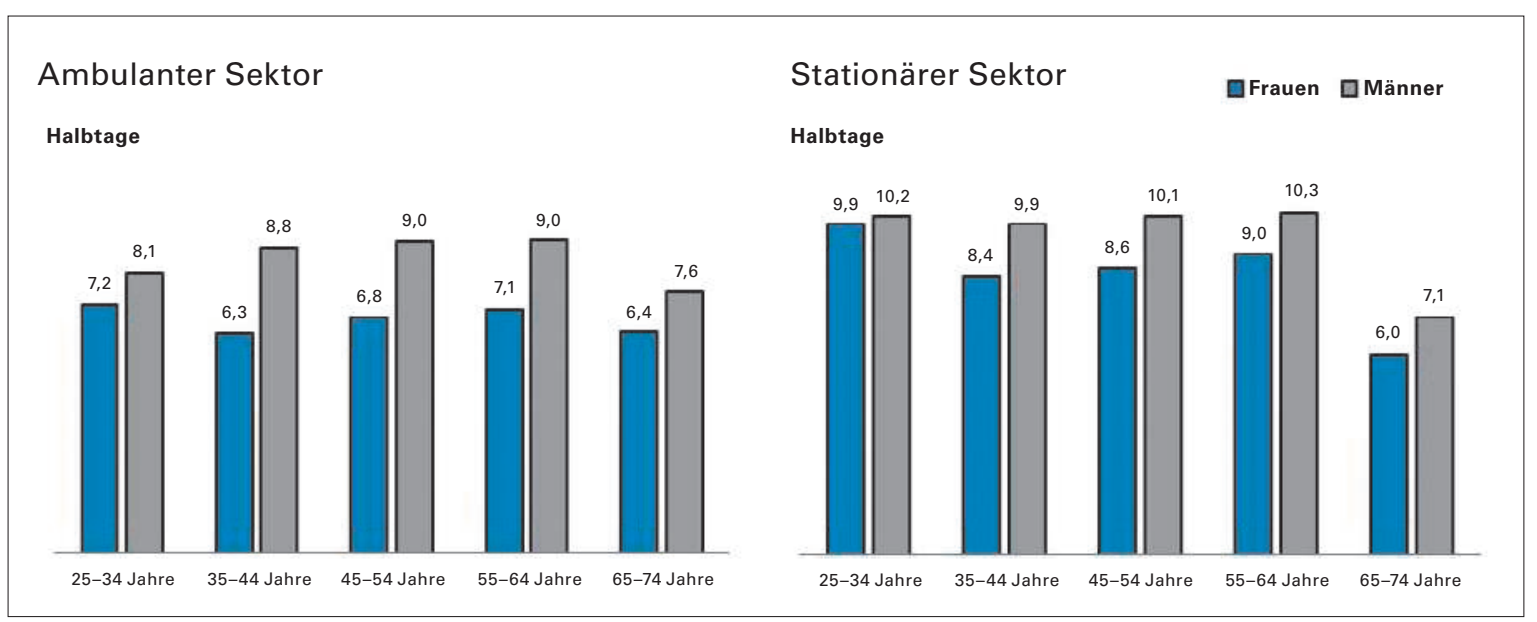

Abbildung 5: Arbeitspensum altersstandardisiert und nach Sektor 2014 ( $n=7295)$.

\section{Arbeitspensum - Anteil Teilzeitarbeitende}

Die Angaben zum Arbeitspensum und alle Auswertungen im nun folgenden zweiten Teil des Artikels basieren auf Daten, welche die Ärzte mittels Umfrage auf dem Mitgliederportal myFMH selbst deklarieren konnten (myFMH-Stichprobe).

Ärztinnen und Ärzte arbeiteten 2014 durchschnittlich 8,9 Halbtage (Tab. 4) pro Woche. Im ambulanten Sektor beträgt das durchschnittliche Arbeitspensum mit 8,2 Halbtagen gut zwei Halbtage weniger als im stationären Bereich (9,4 Halbtage). Das durchschnittliche Arbeitspensum der Ärztinnen (ambulant: 6,8; stationär: 8,8 Halbtage) ist tiefer im Vergleich zu demjenigen ihrer Kollegen (ambulant: 8,9; stationär: 10,1 Halbtage). Seit 2009 nahm das durchschnittliche Arbeitspensum um rund 4 Prozent ab, während im stationären Sektor keine Veränderung beobachtet wurde.

Abbildung 5 zeigt einen geschlechtsspezifischen Unterschied hinsichtlich des Arbeitspensums. Ärztinnen im ambulanten Sektor bevorzugen vermehrt Teilzeitarbeit (Beschäftigungsgrad von rund 60 bis 80 Prozent). Im stationären Bereich ist Teilzeitarbeit

FMH-Ärztestatistik: Ihre Mitarbeit zählt!

Für eine gute Datenqualität ist die FMH auf den Support aller FMH-Mitglieder angewiesen. Das Ausfüllen des Fragebogens oder das Kontrollieren der Daten zur Berufstätigkeit beanspruchen nur wenige Minuten. Helfen Sie mit, die FMH-Ärztestatistik noch aussagkräftiger zu machen. Registrieren oder loggen Sie sich auf dem Mitgliederportal myFMH ein und Sie finden den Fragebogen auf der Startseite. Bei Fragen unterstützen wir Sie gerne: E-Mail: myfmh[at]fmh.ch, Helpline: 031 3591259. weit weniger ausgeprägt. Dies liegt womöglich daran, dass die Weiterbildung der Ärzteschaft vor allem im stationären Bereich erfolgt und während dieser Karrierephase das Arbeitspensum seltener reduziert werden kann.

\section{Praxisstruktur und Ärztenetzwerk}

57,2 Prozent der Ärzte im ambulanten Sektor sind in Einzelpraxen tätig. Dieser Anteil hat seit 2008 um gut 5 Prozent abgenommen. Frauen arbeiten etwa zur Hälfte (48,5 Prozent) in Einzel- oder in Doppelund Gruppenpraxen (51,5 Prozent). Bei den Männern ist der Anteil an in einer Einzelpraxis tätigen Ärzten mit 61,6 Prozent leicht höher. In einer Gruppenpraxis arbeiten durchschnittlich 4, o Ärzte.

Gemäss Selbstdeklaration sind 48,9 Prozent der ambulant tätigen Ärzte einem Ärztenetzwerk angeschlossen. Während 70,9 Prozent der männlichen Kollegen in einem Netzwerk vertreten sind, liegt der Anteil bei ihren Kolleginnen mit 32,8 Prozent deutlich tiefer.

\section{Bedeutung der Daten und Ausblick}

Aus der FMH-Ärztestatistik wird ersichtlich, dass rund ein Drittel (im Fall der Assistenzärzte 44 Prozent) der berufstätigen Ärzteschaft aus dem Ausland stammt. Es ist davon auszugehen, dass das hohe Niveau der Versorgung nur dank den Fachkräften aus dem Ausland aufrechterhalten werden kann. Auf politischer Ebene müssen dringend Massnahmen ergriffen werden, um den Bedarf an Ärzten mit in der Schweiz ausgebildeten Medizinern zu decken. Dies zum einen aus Solidarität zu den Ländern, aus denen die ausländischen Ärzte stammen. Zum anderen weil damit zu 
rechnen ist, dass der mögliche Zustrom an ausländischen Ärzten in die Schweiz auch durch sprachliche Hürden begrenzt wird. Zudem reagieren gewisse Länder wie z.B. Deutschland auf die Abwanderungen ihrer Ärzte und ergreifen Massnahmen, um die Attraktivität der Arbeitsbedingungen in ihrem Land zu steigern bzw. bekannt zu machen.

Die regionalen Unterschiede in der Ärztedichte, der steigende Frauenanteil in der Ärzteschaft und die damit zu erwartende grössere Nachfrage nach Teilzeitarbeit erfordern dringend die Schaffung neuer Arbeitsversorgungsmodelle. Zentral sind hier das verbindliche und koordinierte Vorgehen und die Zusammenarbeit der Leistungserbringer innerhalb eines Fachgebiets, aber auch interdisziplinär und interprofessionell. Unnötige, nicht wirksame Behandlungen und Doppelspurigkeiten vermeiden, Behandlungsprozesse optimieren und vorhandenes Potential ausschöpfen und damit die Behandlungsqualität erhöhen - all diese Punkte sollen handlungsleitende Grundsätze in der künftigen Ausrichtung des Versorgungssystems sein, wie im Bericht Gesundheit 2020 [7] festgehalten wird. Die flächendeckende Führung elektronischer Patientendossiers, verbindliche Behandlungspfade und die Förderung der Gesundheitskompetenz der Patienten stellen dabei wichtige Erfolgsfaktoren dar.
Das Gesundheitssystem ist komplex; viele Einflüsse spielen mit. Um die Auswirkungen und Wirksamkeit von Massnahmen aufzuzeigen und Transparenz zu schaffen, braucht es Messungen und Daten. Die FMHÄrztestatistik leistet einen wichtigen Beitrag zur Bedarfsabklärung und zu weiteren relevanten Fragestellungen. Ohne die Mitarbeit der FMH-Mitglieder wäre dies nicht möglich - die FMH bedankt sich an dieser Stelle für Ihr Engagement und hofft, auch weiterhin auf Ihre Unterstützung zählen zu können.

\section{FMH-Ärztestatistik auf einen Blick}

Interessante Fakten aus der FMH-Statistik 2014 sind attraktiv illustriert und als Poster erhältlich (Deutsch oder Französisch). Zusammengefaltet hat es die handliche Grösse einer Postkarte - das Poster eignet sich also auch bestens zum Auflegen und Mitnehmen. Es würde uns freuen, wenn die FMH-Ärztestatistik im Posterformat in Wartezimmern, Praxis- oder Spitalräumen ihren Platz findet oder an Weiterbildungsveranstaltungen verbreitet wird. Gerne stellen wir Ihnen das Ärztestatistik-Poster in der gewünschten Menge kostenlos zu. Bitte benutzen Sie dafür das Bestellformular auf der FMH-Website (www.fmh.ch $\rightarrow$ Services $\rightarrow$ Statistik $\rightarrow$ Ärztestatistik) oder schreiben eine E-Mail an ddq[at]fmh.ch.

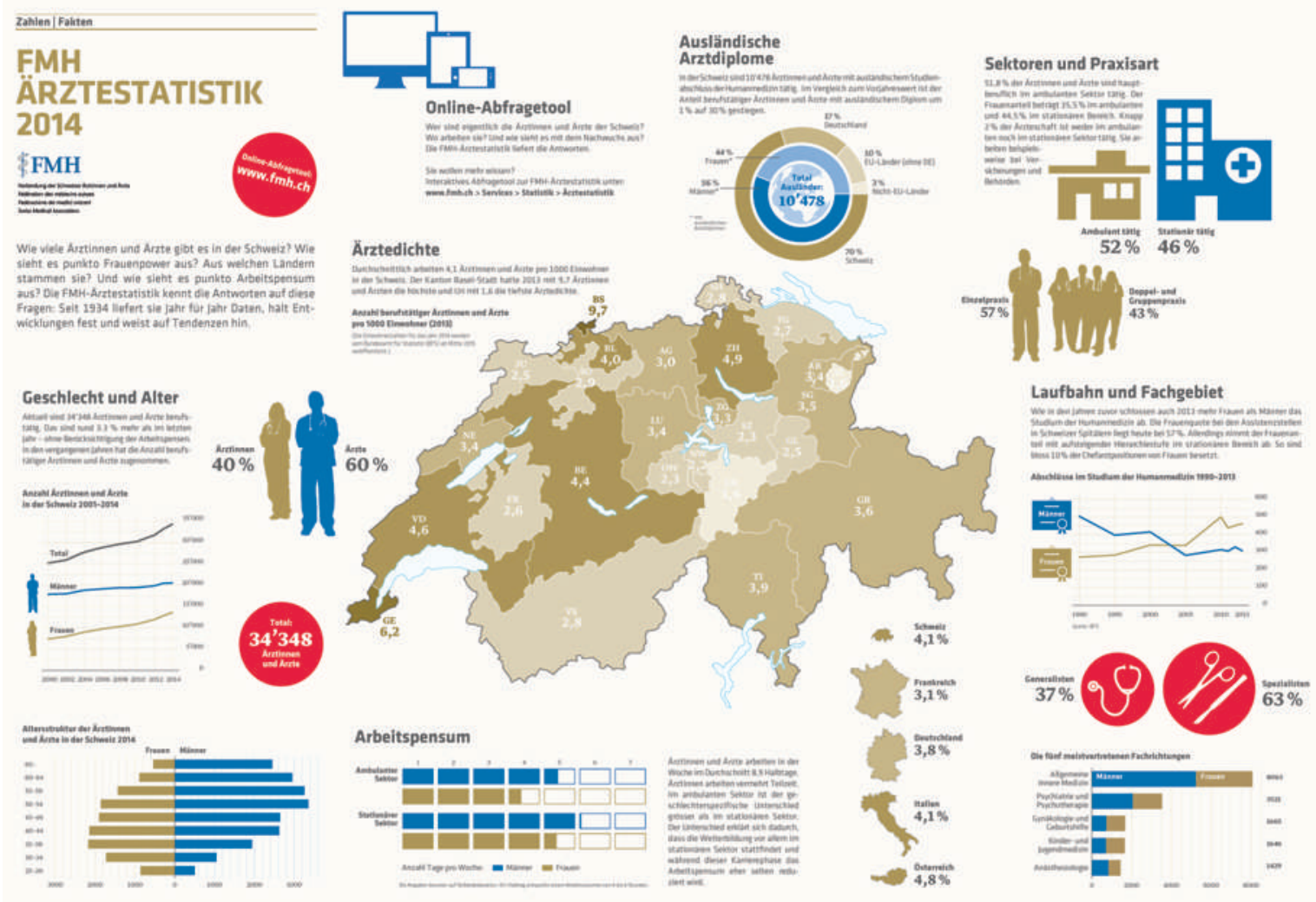




\section{Glossar (alphabetische Reihenfolge)}

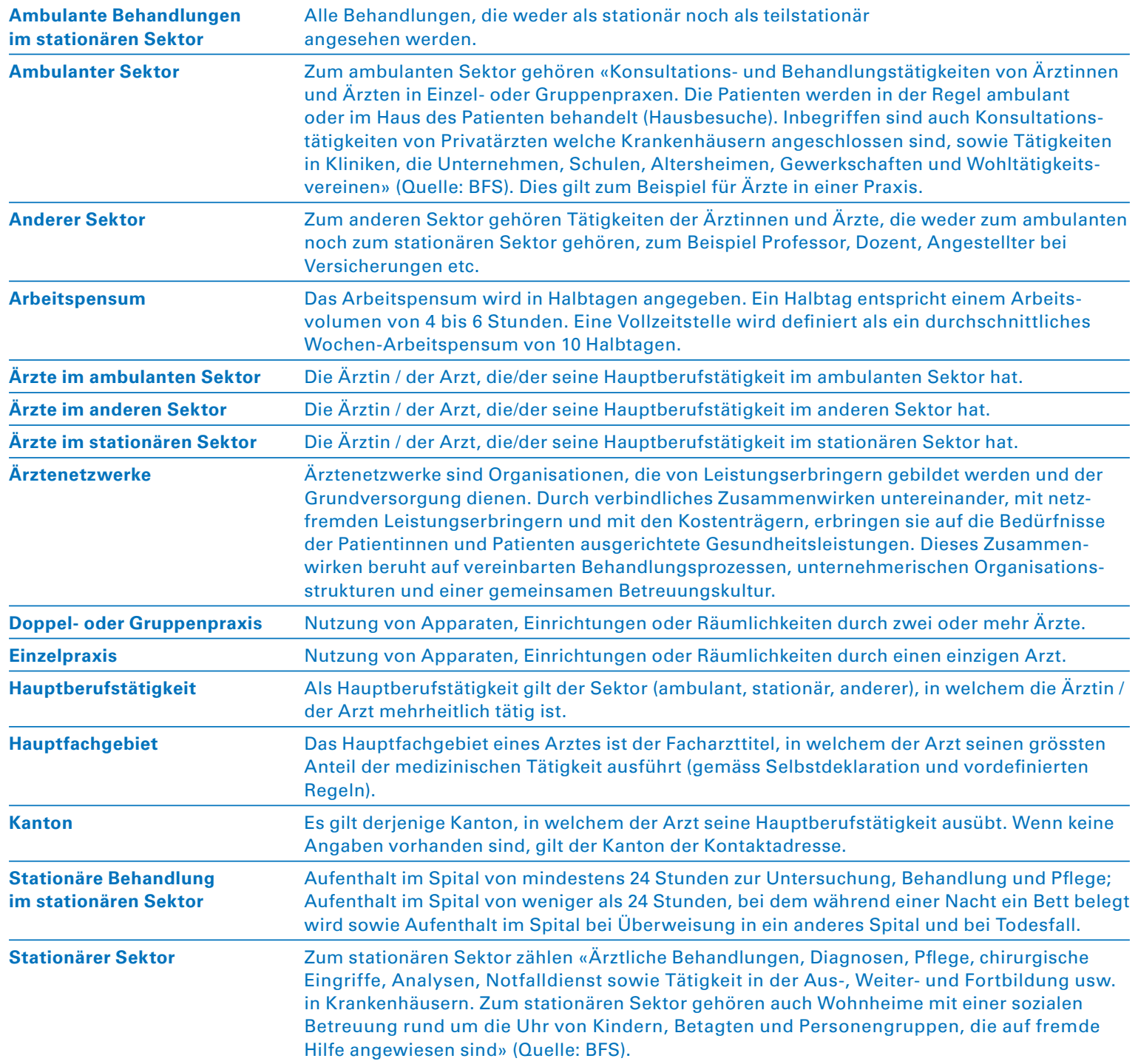

\section{Referenzen}

1 Zur aktuellen Situation der ärztlichen Grundversorgung: www.bag.admin.ch/themen/berufe/13932/13933/14201/?lang=de

2 Dutoit L, Gardiol L, Künzi, K. Etablissement du recensement des médecins actifs en Suisse (Obsan Dossier 28). Neuchâtel: Observatoire suisse de la santé; 2014. Korrespondenz: FMH / Abteilung
Elfenstrasse 18 CH-30oo Bern 15 Tel. 0313591111 www.fmh.ch
3 Hostettler S, Laffranchi R, Kraft E. Ärzteschaft in der SchweizFokus Arbeitspensum. Schweiz Ärztezeitung. 2013;95(50):1891-5.

4 Kraft E, Napierala C. Validierung der Daten für die Versorgungsforschung. Schweiz Ärztezeitung. 2010;91(3):64-6.
5 Humanmedizin Eidgenössische Diplome 2013: www.bag.admin.ch/themen/berufe/13930/13936/14382/index. html?lang=de

6 Länder mit der höchsten Ärztedichte weltweit im Jahr 2013: http://de.statista.com/statistik/daten/studie/232536/umfrage/ laender-mit-der-hoechsten-aerztedichte-weltweit/

7 Eine umfassende Strategie für das Gesundheitswesen: www.bag.admin.ch/gesundheit2o2o/index.html?lang=de 